Preprints of the

Max Planck Institute for

Research on Collective Goods

Bonn 2016/8

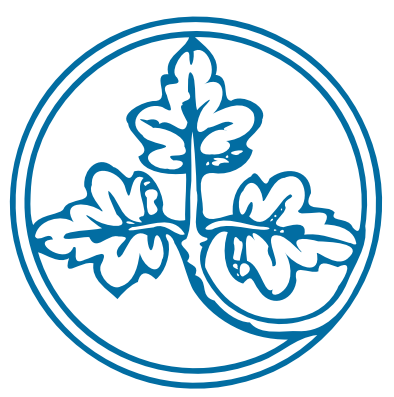

Single versus Multiple

Randomization in

Matching Mechanisms

André Schmelzer

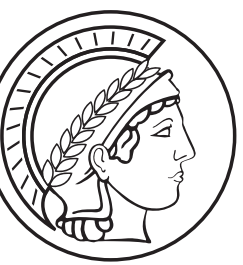




\title{
Single versus Multiple Randomization in Matching Mechanisms
}

\author{
André Schmelzer
}

May 2016

revised version: March 2017 


\title{
Single versus Multiple Randomization in Matching Mechanisms
}

\author{
André Schmelzer*
}

\begin{abstract}
This paper experimentally studies an essential institutional feature of matching markets: Randomization of allocation priorities. I compare single and multiple randomization in the student assignment problem with ties. The Gale-Shapley deferred acceptance algorithm is employed after indifferences in school priorities are resolved by either random procedure. The main result is that a significant fraction of individuals prefers multiple to single randomization, although both are equivalent in expectation. Multiple randomization is perceived to be fairer. One theoretical explanation is the failure to disregard compound lotteries. These results show that random procedures are not inherently neutral with respect to preferences and fairness perceptions.
\end{abstract}

JEL classification: C78, C91, D78, D81

Keywords: market design, school choice, mechanism design, experiment, deferred acceptance algorithm, randomization, tie-breaking.

${ }^{*}$ Max Planck Institute for Research on Collective Goods, Kurt-Schumacher-Str. 10, D-53113 Bonn, Germany. email: schmelzer@coll.mpg.de.

This version: March 2017. First version: May 2016. I thank Dorothea Kübler, Christoph Engel, Rustamdjan Hakimov, Johannes Hemker, Nicolas Roux, Svenja Hippel, Franziska Tausch, Tim Winke, and audiences at the MPI Bonn, WZB Berlin, NYU, IMEBESS Rome, ESA North American meeting Tucson, and SEA Washington D.C. for helpful comments. Financial support from the Max Planck Society for the Advancement of Science is gratefully acknowledged. 


\section{Introduction}

Public resources can be allocated by mechanisms involving lotteries. This approach rests on the assumption that a randomization is fair since everyone has an equal chance to obtain the public resource (Elster, 1991). There is a policy debate on such randomizations in the design of school choice mechanisms. Abdulkadiroğlu et al. $(2005,2009)$ redesigned the New York City (NYC) high school match based on a mechanism involving randomization. Here, randomization is applied if schools are indifferent between students. There are indifference classes and randomization is used within classes. That is, within indifference classes, schools do not admit students based on grades or other criteria, but use lotteries to determine priorities. Two types of randomization are discussed. Indifferences can either be resolved by a single lottery yielding one identical rank order of all students at all schools or by multiple lotteries resulting in a rank order at each school.

The policy debate reveals a tension between social welfare and social acceptability of both types of randomization. Abdulkadiroğlu et al. (2009) find that single randomization is favorable in terms of welfare based on simulations with NYC data. More students receive their top choice under single randomization. This welfare advantage of single randomization is supported by simulations with Amsterdam survey data (De Haan et al., 2016), analytical results (Ashlagi et al., 2015; Arnosti, 2016), and experimental findings (Featherstone and Niederle, 2014). In contrast, NYC policymakers argued that the "equitable approach" would be to employ multiple randomization (Pathak, 2011, p. 523). Single randomization was conjectured to be less socially acceptable. In addition, differences in truthful preference revelation between single and multiple randomization could offset the welfare advantages of single randomization.

Yet, there are no field data systematically comparing single with multiple randomization because only one procedure can be employed at a time. Up to now, we do not know whether and why individuals prefer one type of randomization and whether there is an impact on their truth-telling behavior. I investigate the following research question: Do individuals prefer one random procedure to the other and does the procedure affect their behavior under the mechanism?

This paper examines behavior under the Gale and Shapley (1962) deferred acceptance (DA) algorithm with single and multiple randomization. I employ a con- 
trolled laboratory experiment: While the random procedure is varied, everything else is held constant. I investigate (i) whether individuals prefer either mechanism as well as their driving factors, and whether there is a causal impact of changing the randomization on (ii) truthful preference revelation and (iii) welfare.

The results show that a significant fraction of participants prefers the multiple to the single randomization mechanism while the majority of participants is indifferent. The multiple randomization is found to be perceived fairer using questionnaire data. Consistent with the theory, truthful preference revelation and welfare are not affected by the random procedure to resolve indifferences.

The main contribution of this paper is twofold. First, it tests the causal impact of changing the procedure of randomization on truthful preference revelation while ruling out theoretical welfare differences between the randomizations by design. Second, it provides a behavioral theory and evidence consistent with a strict preference for multiple randomization. The paper shows that randomizations are not per se perceived as fair.

This paper relates to two lines of literature: decision-making involving random procedures and the design of school choice mechanisms. The first part of the research question addresses preferences for random procedures and their driving factors. Keren and Teigen (2010) and Eliaz and Rubinstein (2014) suggest that fairness judgements differ between distinct types of randomization. Bolton et al. (2005) find evidence that the acceptability of unequal outcomes is higher if they are generated by a random procedure. Results from Chlaß et al. (2016) indicate that individuals have procedural preferences, independent of the outcomes. Rubinstein (2002) finds evidence of false diversification in multiple lottery-decision problems. Dwenger et al. (2014) and Agranov and Ortoleva (2015) find evidence consistent with a deliberate preference for randomization. This paper contributes to this literature by providing evidence consistent with a preference for multiple randomization. Moreover, it relates these preferences to fairness judgements.

The second part of the research question relates to the experimental literature on school choice by addressing truthful preference revelation and the resulting welfare effects. School choice experiments employing the DA algorithm were initiated by Chen and Sönmez (2006), following the theoretical work of Abdulkadiroğlu and Sönmez (2003). A number of experimental papers investigate assignment mecha- 
nisms and their sensitivity to specific market design features. For instance, Pais and Pintér (2008) provide evidence that less preference information provided is associated with more truth-telling behavior. Concerning welfare results from the DA algorithm, Calsamiglia et al. (2010) find that constraining the number of schools in preference rankings can have a negative impact on efficiency. Departing from previous work, this paper is the first to focus on the random procedure resolving indifferences in allocation priorities prior to applying the DA algorithm. The novelty of the experimental design is that preference rankings under both random procedures are obtained and that subjects are asked to state which procedure they prefer to participate in. This paper contributes to the school choice literature by investigating the impact of the randomization design on truthful preference revelation and the resulting welfare.

The remainder of the paper is organised as follows. Section 2 introduces the DA algorithm and its properties under randomization, a behavioral theory consistent with a preference for multiple randomization, and the predictions for the experiment. The experimental design and procedure is described in section 3. Section 4 provides the main results that a significant fraction of individuals prefers multiple to single randomization while the majority is indifferent, and that the amount of truth-telling does not differ between the two mechanisms. Section 5 concludes. 


\section{Theoretical framework}

\subsection{Deferred acceptance algorithm with randomization}

Consider the problem of assigning indivisible goods to individuals without monetary transfers. I focus on a stylized school choice problem following Abdulkadiroğlu et al. (2009). A set of students is assigned to a set of schools according to student preferences and strict school priorities or indifferences. School priorities are exogenous. Each school has the capacity of one seat.

The student assignment variant of the Gale and Shapley (1962) studentproposing DA algorithm with prior randomization works as follows (adapted from Abdulkadiroğlu and Sönmez, 2003). At first, schools may have strict priorities or may be indifferent between students. Students submit strict preference rankings over schools.

- If the school is indifferent between students, its priorities are determined randomly from a uniform distribution.

- Step 1: Students apply to the school at the top of their submitted ranking. One student is tentatively accepted at each school according to her priority. All others are rejected.

- Step $k, k \geq 2$ : Rejected students apply to their next preferred school. Tentatively accepted and new applicants are jointly considered. The seat is tentatively assigned based on the priority and all unassigned students are rejected.

- Stop the algorithm if no student is rejected anymore.

When the algorithm terminates, the tentative turns into a final matching.

Strict student preferences and strict school priorities are a necessary condition of the DA algorithm (Gale and Shapley, 1962). In case of schools being indifferent between students, a strict priority ordering is obtained by randomization. I consider two random procedures to resolve indifferences at schools: Single and multiple randomization. Under single randomization, one single lottery is employed to obtain 
one rank order of all students. This ranking is used to break ties at schools. Thereafter, the DA algorithm is applied. Under multiple randomization, school-specific lotteries are employed. Each school applies an independent lottery and thereby obtains its own priority order of students before running the DA algorithm.

\subsection{Properties of the deferred acceptance algorithm with randomization}

This section summarizes the theoretical properties of the student-proposing DA with random tie-breaking of priorities. In line with the literature, I consider the desirable properties stability, strategy-proofness, and Pareto efficiency for students.

Stability. The DA algorithm is stable and student-optimal under strict student preferences and school priorities (Gale and Shapley, 1962). That is, there is no blocking pair of a school and a student, such that the school prefers the student to an assigned student and the student prefers the school to her actually matched school. The stability property holds independent of the random procedure to break ties.

Strategy-proofness. The student-proposing DA algorithm is strategy-proof for students (Dubins and Freedman, 1981; Roth, 1982). That is, truthful preference revelation is a dominant strategy for every student, independent of the preferences of the other students. Abdulkadiroğlu et al. (2009) show that strategy-proofness holds independent of the random procedure. They show that if the dominant strategy incentive compatibility holds for every student and for every priority and preference profile, then the DA is strategy proof for any arbitrary tie-breaking rule yielding a priority profile. The dominant strategy equilibrium of the preference revelation game is not changed by the tie-breaking.

Efficiency. The matching of the DA algorithm is efficient if there is no other matching outcome dominating it. In general, the DA algorithm is not Pareto efficient when considering student welfare only (Roth, 1982). However, the DA algorithm under strict priority and preference profiles yields the student-optimal stable matching. It is constrained efficient. Every student prefers the DA matching outcome to any other stable outcome (Gale and Shapley, 1962).

Tie-breaking can negatively affect efficiency. The DA algorithm with random 
tie-breaking is not ex post constrained efficient (Erdil and Ergin, 2008). Abdulkadiroğlu and Sönmez (2003) first discussed the additional efficiency loss due to exogenous randomization of originally indifferent priorities.

Welfare can differ between tie-breaking rules. Single randomization can be shown to have superior welfare properties depending on the environment. However, Abdulkadiroğlu et al. (2009) show that single and multiple randomization cannot be compared by stochastic dominance. Arnosti (2016) and Ashlagi et al. (2015) analytically show that multiple randomization produces fewer matchings with the top choice compared to single randomization. Simulations of Ashlagi et al. (2015) also indicate that single randomization allocates more students to the upper end of the expected rank distribution. In general, matching distributions under single and multiple randomization are sensitive to the setting in terms of capacities, market size, preference lists (Ashlagi et al., 2015), and supply/demand balance (Ashlagi and Nikzad, 2015).

\subsection{Efficiency in the experiment}

I construct an environment such that constrained efficiency does not differ between matching distributions produced under single and multiple randomization assuming truthful preference revelation. To see this, consider the assignment problem in the experiment with four students $i_{1}, i_{2}, i_{3}, i_{4}$, and four schools $s_{1}$, $s_{2}, s_{3}, s_{4}$, where each school is providing one seat. I construct the following student preference profiles over schools $P_{i}=\left\{P_{i_{1}}, \ldots, P_{i_{4}}\right\}$ such that the matching distributions do not depend on the randomization procedure:

$$
\begin{array}{lllllll}
s_{1} & P_{i_{1}} & s_{2} & P_{i_{1}} & s_{3} & P_{i_{1}} & s_{4} \\
s_{1} & P_{i_{2}} & s_{2} & P_{i_{2}} & s_{3} & P_{i_{2}} & s_{4} \\
s_{1} & P_{i_{3}} & s_{4} & P_{i_{3}} & s_{3} & P_{i_{3}} & s_{2} \\
s_{1} & P_{i_{4}} & s_{4} & P_{i_{4}} & s_{3} & P_{i_{4}} & s_{2}
\end{array}
$$

The students $i_{1}, i_{2}$, and $i_{3}, i_{4}$ form pairs with identical preferences. 
Motivating example. The original school priorities over students $R_{s}=$ $\left\{R_{s_{1}}, \ldots, R_{s_{4}}\right\}$ are strict $\succ_{s}$ for school 4 only. Schools $s_{1}, s_{2}$, and $s_{3}$ are originally indifferent $\sim_{s}$. Randomization induces strict priorities at the first three schools.

Assume the following strict priorities from single randomization:

$$
\begin{aligned}
& i_{1} \succ_{s_{1}} i_{2} \succ_{s_{1}} i_{3} \succ_{s_{1}} i_{4} \\
& i_{1} \succ_{s_{2}} i_{2} \succ_{s_{2}} i_{3} \succ_{s_{2}} i_{4} \\
& i_{1} \succ_{s_{3}} i_{2} \succ_{s_{3}} i_{3} \succ_{s_{3}} i_{4} \\
& i_{1} \succ_{s_{4}} i_{2} \succ_{s_{4}} i_{3} \succ_{s_{4}} i_{4}
\end{aligned}
$$

The resulting student-optimal stable matching under single randomization is indicated by the boxes:

$$
\begin{array}{lllllll}
s_{1} & P_{i_{1}} & s_{2} & P_{i_{1}} & s_{3} & P_{i_{1}} & s_{4} \\
s_{1} & P_{i_{2}} & s_{2} & P_{i_{2}} & s_{3} & P_{i_{2}} & s_{4} \\
s_{1} & P_{i_{3}} & s_{4} & P_{i_{3}} & s_{3} & P_{i_{3}} & s_{2} \\
s_{1} & P_{i_{4}} & s_{4} & P_{i_{4}} & s_{3} & P_{i_{4}} & s_{2}
\end{array}
$$

Assume three examples of multiple randomizations:

$$
\begin{aligned}
& i_{1} \succ_{s_{1}} i_{2} \succ_{s_{1}} i_{3} \succ_{s_{1}} i_{4} \quad i_{3} \succ_{s_{1}} i_{4} \succ_{s_{1}} i_{2} \succ_{s_{1}} i_{1} \quad i_{2} \succ_{s_{1}} i_{3} \succ_{s_{1}} i_{1} \succ_{s_{1}} i_{4} \\
& i_{4} \succ_{s_{2}} i_{3} \succ_{s_{2}} i_{1} \succ_{s_{2}} i_{2} \quad i_{2} \succ_{s_{2}} i_{1} \succ_{s_{2}} i_{3} \succ_{s_{2}} i_{4} \quad i_{4} \succ_{s_{2}} i_{3} \succ_{s_{2}} i_{1} \succ_{s_{2}} i_{2} \\
& i_{1} \succ_{s_{3}} i_{2} \succ_{s_{3}} i_{4} \succ_{s_{3}} i_{3}, \quad i_{4} \succ_{s_{3}} i_{3} \succ_{s_{3}} i_{2} \succ_{s_{3}} i_{1}, \quad i_{1} \succ_{s_{3}} i_{2} \succ_{s_{3}} i_{4} \succ_{s_{3}} i_{3} \\
& i_{1} \succ_{s_{4}} i_{2} \succ_{s_{4}} i_{3} \succ_{s_{4}} i_{4} \quad i_{1} \succ_{s_{4}} i_{2} \succ_{s_{4}} i_{3} \succ_{s_{4}} i_{4} \quad i_{1} \succ_{s_{4}} i_{2} \succ_{s_{4}} i_{3} \succ_{s_{4}} i_{4}
\end{aligned}
$$

The resulting matchings (box) from the DA under each multiple randomization are

$$
\begin{aligned}
& \begin{array}{|lllllllllllllllllllllllllllllllll}
s_{1} & P_{i_{1}} & s_{2} & P_{i_{1}} & s_{3} & P_{i_{1}} & s_{4} & s_{1} & P_{i_{1}} & s_{2} & P_{i_{1}} & s_{3} & P_{i_{1}} & s_{4} & s_{1} & P_{i_{1}} & s_{2} & P_{i_{1}} & s_{3} & P_{i_{1}} & s_{4}
\end{array} \\
& \begin{array}{llllllll|lllllllllllllllllllllllll}
s_{1} & P_{i_{2}} & s_{2} & P_{i_{2}} & s_{3} & P_{i_{2}} & s_{4} & s_{1} & P_{i_{2}} & s_{2} & P_{i_{2}} & s_{3} & P_{i_{2}} & s_{4} & s_{i_{2}} & s_{2} & P_{i_{2}} & s_{3} & P_{i_{2}} & s_{4}
\end{array} \\
& \begin{array}{llllllllllllllllllllllll}
s_{1} & P_{i_{3}} & s_{4} & P_{i_{3}} & s_{3} & P_{i_{3}} & s_{2} & s_{1} & P_{i_{3}} s_{4} & P_{i_{3}} & s_{3} & P_{i_{3}} & s_{2} & s_{1} & P_{i_{3}} & s_{4} & P_{i_{3}} & s_{3} & P_{i_{3}} & s_{2}
\end{array} \\
& \begin{array}{lllllllllllllllllllllllllllllllllll}
s_{1} & P_{i_{4}} & s_{4} & P_{i_{4}} & s_{3} & P_{i_{4}} & s_{2} & s_{1} & P_{i_{4}} & s_{4} & P_{i_{4}} & s_{3} & P_{i_{4}} & s_{2} & s_{1} & P_{i_{4}} & s_{4} & P_{i_{4}} & s_{3} & P_{i_{4}} & s_{2}
\end{array}
\end{aligned}
$$

The matching distributions from the DA do not differ between single and multiple randomization. This result is generalized for the given $P_{i}$ in Proposition 1. 
Proposition 1. Given the preference relations $P_{i}$ in the experiment, one student gets into her top choice, two students get into their second preference, and one student gets into her third preference. This holds irrespective of the priority relations $R_{s_{1}}, R_{s_{2}}$, and $R_{s_{3}}$. The distribution of outcomes does not depend on the randomization procedure.

Sketch of proof. To see that Proposition 1 holds, assume truthful preference revelation of $P_{i}$, fix the priority relation $R_{s_{4}}$, and consider the priority relations $R_{s_{1}}$, $R_{s_{2}}$, and $R_{s_{3}}$ produced by single and multiple randomization. Note that each pair of students has identical preference profiles. In this setting, the student-proposing DA algorithm produces the final matching in three steps.

Single randomization. The priority relations are identical: $R_{s_{1}}=R_{s_{2}}=R_{s_{3}}$. In step 1 of the DA, all students apply to their top choice $s_{1}$ and one student is accepted. In step 2, the three rejected students apply to their second preference, which is either $s_{2}$ or $s_{4}$. The student from the pair where the second student was accepted at $s_{1}$ in step 1 is accepted at her second preference $\left(s_{2}\right.$ or $\left.s_{4}\right)$. I call the pair that is jointly applying to their second preference the remaining pair. For the remaining pair, the priority relation of either $R_{s_{2}}$ or $R_{s_{4}}$ determines who is accepted. The accepted student gets into her second preference and the rejected student gets into her third preference $s_{3}$ (after applying in step 3). Every student gets at least into her third preference. Importantly, it follows from the above argument that the fourth preference of each student and $R_{s_{3}}$ are irrelevant for determining the final matching of the DA under truth-telling.

Multiple randomization. The priority relations $R_{s_{1}}, R_{s_{2}}$, and $R_{s_{3}}$ may be identical, if the three lotteries yield identical outcomes (as in the single randomization). Consider the subset of priorities where $R_{s_{1}} \neq R_{s_{2}} \neq R_{s_{3}}$. Since $R_{s_{3}}$ is irrelevant for determining the final matching, the relevant subset of priorities reduces to $R_{s_{1}} \neq R_{s_{2}}$. $R_{s_{2}}$ determines who is accepted at her second preference. This holds for both cases $R_{s_{1}} \neq R_{s_{2}}$ and $R_{s_{1}}=R_{s_{2}}$ when students submit their true preferences. 


\subsection{Preference for multiple randomization}

A policymaker argued during the discussion about the NYC high school match:

"I believe that the equitable approach is for a child to have a new chance with each program. [...] If we use only one random number, and I had the bad luck to be the last student in the line this would be repeated 12 times and I would never get a chance" (Pathak, 2011, p. 523).

The behavioral theory in this section accommodates choices consistent with this preference for multiple randomization. It provides structure for the choice problem.

Definitions. Let the set of students be $I=\{1, \ldots, N\}$ and the set of schools be $S=\left\{s_{1}, \ldots, s_{M}\right\}$ with the generic element $s_{m}$, where each school has one seat capacity. Students have strict preferences $P_{i}=\left\{P_{i_{1}}, \ldots, P_{i_{N}}\right\}$. Schools have priorities $R_{s}=\left\{R_{s_{1}}, \ldots, R_{s_{M}}\right\}$ which are strict $\succ_{s}$ or marked by indifference $\sim_{s}$. Let the single randomization be a simple lottery tie-breaking rule and multiple randomization be a compound lottery. In general, let $L=\left(p_{1}, \ldots, p_{M}\right)$ be the simple lottery over the possible material payoffs $\pi_{i, s_{m}}$ from the DA algorithm. The payoff $\pi_{i, s_{m}}: S \rightarrow \mathbb{R}$ of student $i$ is obtained with probability $p_{m} \in[0,1]$, with $\sum_{m} p_{m}=1$. Let $\mathcal{L}=\left\{L_{s_{1}}, \ldots, L_{s_{M}} ; \alpha_{1}, \ldots, \alpha_{M}\right\}$ be the compound lottery yielding the simple lottery $L_{s_{m}}$ with probability $\alpha_{m} \geq 0$ for $m=1, \ldots, M$.

Figure 1 illustrates the compound lottery structure implied by the DA algorithm: The second lottery is only relevant if the first lottery is lost in expectation.

Compound lottery $(\mathcal{L})$

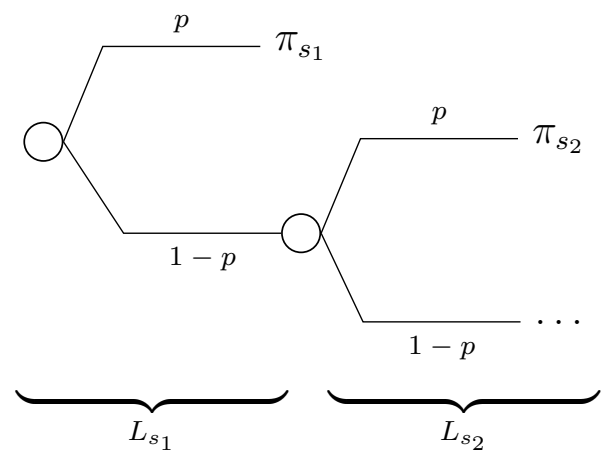

Figure 1: Tree representation of a two-stage compound lottery. 
Expected utility theory. The reduction of the compound lottery under expected utility theory $(\mathrm{EU})$ is considered as the benchmark. The following example illustrates the choice problem in the experiment mapping its environment of four students and four schools. Let $L=\left(q_{1}, \ldots, q_{4}\right)$ be the simple lottery over the payoffs $\pi_{i, s_{m}}$ from the DA. Let $\mathcal{L}=\left\{L_{s_{1}}, \ldots, L_{s_{4}} ; \alpha_{1}, \ldots, \alpha_{4}\right\}$ be the four-stage compound lottery of the four simple lotteries $L_{s_{m}}=\left(q_{1}^{s_{m}}, \ldots, q_{4}^{s_{m}}\right), m=1, \ldots, 4$, over payoffs at the schools $s_{1}, s_{2}, s_{3}$, and $s_{4}$. The degenerate lottery $L_{s_{4}}$ yields the payoff with certainty. Let the preference relation satisfy the standard axioms of EU. Assume truthful preference revelation. The consequentialist assumption states that individuals only care about the reduced lottery. Proposition 2 implies that the individuals are indifferent between the simple and the compound lottery (proof in Appendix B). This result holds irrespective of the functional form of the utility function $U$.

Proposition 2. The expected utility of the simple lottery $L$ is equivalent to the expected utility of the compound lottery $\mathcal{L}, E U(L)=E U(\mathcal{L})$.

Standard EU predicts that single and multiple randomization are equivalent in expectation with respect to receiving utility from the payoff of the DA algorithm.

Behavioral theory. The following behavioral framework is based on the nonconsequentialist assumption that individuals do not consider the outcomes of the DA algorithm, but the ranks from the lotteries. This implies a cognitive failure to grasp the compound lottery structure. Since the distribution of matching outcomes does not depend on the randomization procedure, the shape of the utility function cannot explain the preference for multiple randomization under standard EU.

I propose that individuals do not evaluate the outcome distribution of payoffs from the DA algorithm, but rather that they evaluate individual lotteries in terms of their position in the priority ranking. They care about their position "in the line" (Pathak, 2011, p. 523). If this behavioral assumption is satisfied, then the concavity of the utility function is a sufficient condition to explain a preference for multiple randomization. In principle, risk or loss aversion suffice for concavity. Here, I show the result for loss aversion. 
In order to accommodate choices that are consistent with a preference for multiple randomization, let the behavioral assumption be satisfied. Let the utility function $f$ be defined over the set of ranking positions $\left(k_{s_{1}}, k_{s_{2}}\right)$ :

A0. $f:\left(k_{s_{1}}, k_{s_{2}}\right) \rightarrow \mathbb{R}$, with $f\left(k_{s_{1}}, k_{s_{2}}\right)=k_{s_{1}}+k_{s_{2}}$ and $f\left(k_{s_{1}}, k_{s_{2}}\right)>f\left(k_{s_{1}}^{\prime}, k_{s_{2}}\right)$ if $k_{s_{1}}>k_{s_{1}}^{\prime}$.

$k_{s_{1}}$ is the position in the ranking at school 1 and $k_{s_{2}}$ is the ranking position at school 2, with $k_{s_{m}} \in\{x, y\}$. Let the first position yield $x$ and the second position yield $y$, with $x>y$, where the gain-loss utility function $\mu$ is defined over these outcomes from the ranking.

Let the following assumptions from Köszegi and Rabin (2007) about the gain-loss utility function $\mu$ be satisfied:

A1. $\mu(x)$ is continuous $\forall x$, twice differentiable for $x \neq 0$, and $\mu(0)=0$.

A2. $\mu(x)$ is strictly increasing.

A3. If $y>x \geq 0$, then $\mu(y)+\mu(-y)<\mu(x)+\mu(-x)$.

A4. $\forall x \neq 0, \mu^{\prime \prime}(x)=0$.

A5. $\mu_{-}^{\prime}(0) / \mu_{+}^{\prime}(0) \equiv \lambda>1, \mu_{+}^{\prime}(0) \equiv \lim _{x \rightarrow 0} \mu^{\prime}(|x|)$ and $\mu_{-}^{\prime}(0) \equiv \lim _{x \rightarrow 0} \mu^{\prime}(-|x|)$.

I call assumption $A 0$ the unconditional lottery evaluation, as opposed to the reduction of compound lotteries. This assumption implies that the compound lottery implied by the DA algorithm is disregarded. Instead, the lottery at each school is evaluated unconditionally in terms of ranking positions. As a result, individuals have preferences over lotteries over a set of rankings.

Assumptions $A 1, A 2, A 3$, and $A 5$ are the Kahneman and Tversky (1979) prospect theory properties of the utility function as originally formulated in Bowman et al. (1999, p. 157). ${ }^{1} A 3$ and $A 5$ imply loss aversion $(\lambda)$.

Figure 2 presents the tree representation of both randomizations under $A 0$ in the two lottery case. It illustrates Proposition 3. Single randomization yields the same ranking distribution at both schools, $(x ; x)$ or $(y ; y)$, with one random draw.

\footnotetext{
${ }^{1}$ Assumption $A 4$ ( $A 3^{\prime}$ in Köszegi and Rabin (2007)) excludes diminishing sensitivity to gains and losses. Linearity is the parsimonious and empirically plausible assumption in the lottery outcome space of the school choice problem.
} 

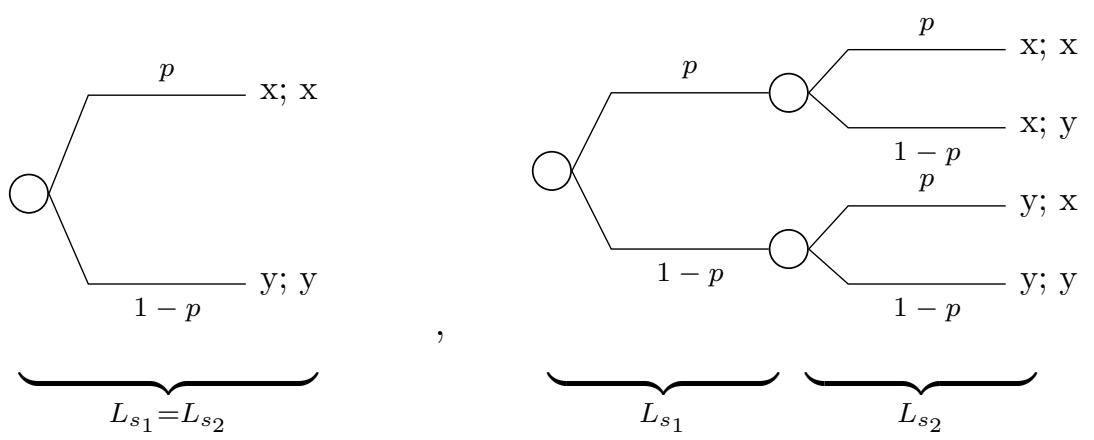

Figure 2: Tree representation of single and multiple randomization under $A 0$.

Under single randomization, the probability of being "the last student in the line" (Pathak, 2011, p. 523) ( $y ; y)$ is $1-p$. Under multiple randomization, the joint probability of running the misfortune of getting the second rank at each school $(y ; y)$ is $(1-p)^{2}$. In contrast to single, the multiple randomization yields mixed ranking outcomes $(x ; y)$ and $(y ; x)$. Mixed ranking outcomes imply a "new chance" (Pathak, 2011, p. 523) distinct from the compound structure (see Figure 1).

Proposition 3. Assuming $A 0-A 5$, the multiple randomization $M L$ is strictly preferred to the single randomization $L, M L \succ L$.

Proof. Consider the utility function $U$ under single $(L)$ and multiple $(M L)$ randomization in the two lottery case:

$$
U(L)<U(M L)
$$

By $A 0$ :

$$
\begin{aligned}
\Leftrightarrow & p \mu(2 x)+(1-p) \mu(2 y) \\
& <p^{2} \mu(2 x)+p(1-p) \mu(x+y)+(1-p) p \mu(y+x)+(1-p)^{2} \mu(2 y) \\
\Leftrightarrow & \left(p-p^{2}\right) \mu(2 x)+\left[(1-p)-(1-p)^{2}\right] \mu(2 y)-2 p(1-p) \mu(x+y)>0 \\
\Leftrightarrow & \left(p-p^{2}\right) \mu(2 x)+\left(p-p^{2}\right) \mu(2 y)-2 p(1-p) \mu(x+y)>0 \\
\Leftrightarrow & \mu(2 y)+\mu(2 x)<2 \mu(x+y) .
\end{aligned}
$$

By assumptions $A 3$ and $A 5$, equation (1) holds. 


\subsection{Predictions}

The experimental hypotheses are based on matching theory and the behavioral theory on preferences for procedures. First, consider the main hypothesis on preference for multiple randomization. The null hypothesis on preferences follows from equivalence of both random procedures in Proposition 2. Under standard EU assumptions, individuals are predicted to be indifferent between the simple lottery $L$ and the compound lottery $\mathcal{L}, L \sim \mathcal{L}$.

By Proposition 3, individuals strictly prefer the multiple lotteries $M L$ to the single lottery $L$ under unconditional lottery evaluation, $L \prec M L$.

Hypothesis 1. (Preference for multiple randomization) Individuals prefer the multiple randomization to the single randomization.

Since both mechanisms are strategy-proof, truth-telling behavior is not predicted to differ. The dominant strategy equilibrium is not changed by the random procedure to break ties (Abdulkadiroğlu et al., 2009). As a result, individuals are predicted to submit the same induced preference rankings under both mechanisms.

Hypothesis 2. (Truthful preference revelation) Individuals truthfully reveal their preferences for schools.

Truthful preference revelation implies that the proportions of truth-telling are identical under the single and the multiple randomization mechanism.

By Proposition 1, the single randomization and multiple randomization are predicted to yield equal outcome distributions. Only student welfare is considered.

Hypothesis 3. (Welfare) Constrained efficiency does not differ between the DA outcomes obtained under the single and the multiple randomization.

Theoretical results on student welfare critically rely on truthful preference revelation. That is, Hypothesis 3 depends on the validity of Hypothesis 2. 


\section{Experimental design}

Predictions are investigated in a laboratory experiment. Instructions are available in Appendix C. ${ }^{2}$ The experiment is designed to test differences in preferences for random procedures and truth-telling behavior in assignment mechanisms with single $(S R)$ and multiple $(M R)$ randomization. Both assignment mechanisms are based on the DA algorithm. They differ only in one aspect: The randomization to obtain strict school priorities before applying the DA algorithm.

- In mechanism SR, one single lottery is employed to obtain one rank order of all students. This ranking is used to break ties at schools.

- In mechanism MR, three lotteries are employed. Each school breaks ties with an independent lottery and obtains its own priority order of students.

\subsection{Environment}

The stylized assignment problem in this experiment consists of a set of four students $I=\left\{i_{1}, i_{2}, i_{3}, i_{4}\right\}$ and a set of four schools $S=\left\{s_{1}, s_{2}, s_{3}, s_{4}\right\}$, where each school has one seat capacity. The designed environment comprises the following strict student preference profiles $P_{i}$ and exogenously given school priorities $R_{s}$. Priorities may be strict $\succ_{s}$ or indifference $\sim_{s}$ :

$$
\begin{aligned}
& s_{1} P_{i_{1}} s_{2} \quad P_{i_{1}} s_{3} \quad P_{i_{1}} s_{4} \quad i_{1} \sim_{s_{1}} i_{2} \sim_{s_{1}} i_{3} \sim_{s_{1}} i_{4}
\end{aligned}
$$

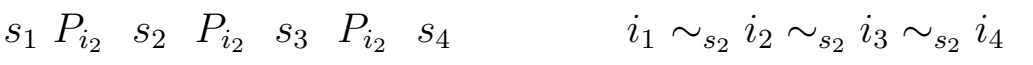

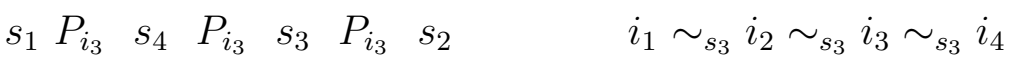

$$
\begin{aligned}
& \begin{array}{llllllll}
s_{1} & P_{i_{4}} & s_{4} & P_{i_{4}} & s_{3} & P_{i_{4}} & s_{2}
\end{array} \quad, \quad i_{1} \succ_{s_{4}} i_{2} \succ_{s_{4}} i_{3} \succ_{s_{4}} i_{4}
\end{aligned}
$$

Induced preferences are correlated. For instance, students 1 and 2 prefer being matched to school 1 over school 2, school 2 over school 3, and school 3 over school 4. Each student is matched to one school only. Participants earn points during the experiment, which are transferred at a rate of $1 / 20$ to euros. Payoffs are symmetric. If participants obtain their top school match, they are paid 14 euros. They receive 10 euros for their second-best, 6 euros for their third-best match, and 2

\footnotetext{
${ }^{2}$ The school choice problem in the experiment is framed as a market with "applicants" applying for "positions" (see also Klijn et al., 2013; Pais and Pintér, 2008). The advantage is that payments have a straightforward interpretation as salaries. See Appendix C for instructions.
} 
euros for their match with the last school. Schools are not strategic players. Only school 4 has strict preferences ex ante. ${ }^{3}$ The first three schools are indifferent between students. In the experiment, mechanisms $S R$ and $M R$ differ in their random procedure to resolve these indifferences. Importantly, the lotteries to resolve indifferences in school priorities are not realized until the end of the experiment.

\subsection{Setup}

Figure 3 provides an overview of the experimental setup consisting of four parts. The experiment implements a one-shot game with complete information. In $S R-M R$, participants first submit their preference ranking for the assignment mechanism $S R$ and then for $M R$. This order is reversed in $M R-S R$. In the beginning, individuals are randomly matched in groups of four and assigned to the $S R-M R$ or the $M R-S R$ conditions. Then, each participant is randomly assigned the role of a student within each group. Roles remain constant.
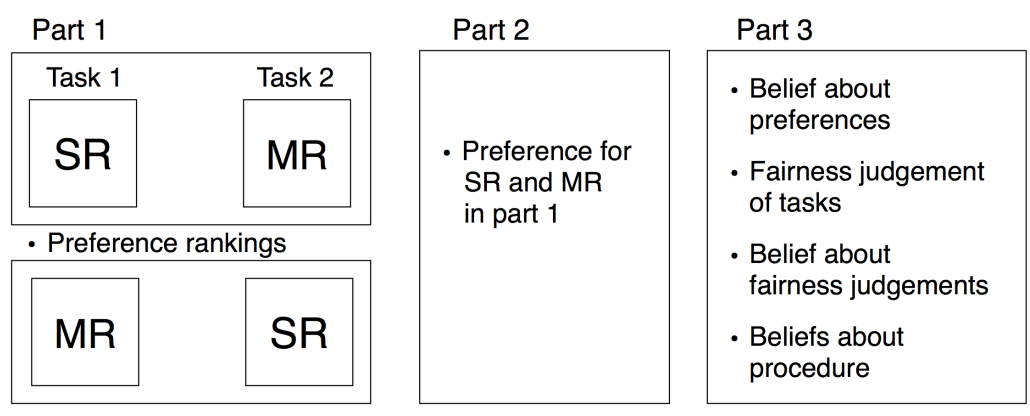

\begin{tabular}{|l|} 
Part 4 \\
\hline Payment: \\
Part 1: randomly \\
either SR or MR \\
Part 2: additionally \\
one randomly \\
selected participant \\
per group
\end{tabular}

Figure 3: Experimental setup. Mechanisms: $(S R)$ Single randomization and DA; $(M R)$ multiple randomization and DA.

Part 1. Part one consists of two tasks: submitting preference rankings under single and multiple randomization. Participants start with task 1 by submitting their first preference ranking to apply to schools. Subjects know from the beginning that they face two different tasks where they submit preference rankings over schools. They

\footnotetext{
${ }^{3}$ Allowing for schools with strict priorities and schools with indifferences at the same time constitutes a generalization of the canonical school choice model in Abdulkadiroğlu and Sönmez (2003). If all schools are indifferent, this reduces to a version of the random priority mechanism (Pathak and Sethuraman, 2011).
} 
also know that only one - either task 1 or task 2 - will be randomly determined to be payoff-relevant. However, they get to know the second task only after they have submitted their preference ranking in the first task. In task 2, participants submit the school ranking for the second mechanism.

Part 2. The preferences over the procedures in part 1 are elicited (without any announcement). Subjects may pay ten euro cents to choose between $S R$ and $M R$. That is, they choose which of the procedures, from the first part of the experiment, should be relevant in determining their payoffs. If they do not pay to make an active choice, the computer randomly draws one of the two with equal probability. ${ }^{4}$

Part 3. Fairness judgements about either mechanism are elicited subsequently on a seven-point Likert scale. Fairness beliefs about the average fairness judgement of the other participants are elicited with a monetary incentive. Further, incentivized beliefs about the chance of getting the high payoff in one procedure, and incentivized beliefs about the average preferences of the other participants are elicited. All incentivations are based on point estimates with a payment of 0.5 euros for correct answers. The questionnaire contains a risk attitude measurement, as in Holt and Laury (2002), as well as questions on the demographic characteristics. ${ }^{5}$

Part 4. After the experiment, the uncertainty is resolved by playing the lotteries and running the DA for each matching group. Subjects are paid anonymously and privately. Everyone gets paid for part 1. In part 1, it is randomly determined for each subject individually whether she gets paid for $S R$ or $M R$. Additionally, one randomly drawn participant of each group is paid for part 2 .

\subsection{Procedure}

Experimental sessions were conducted at BonnEconLab in December 2015 and January 2016. In total, $N=192$ participants took part in the experiment. About $48 \%$ of them were female (in both conditions). Participants were recruited from the BonnEconLab subject pool (more than 6000 subjects) using hroot (Bock et al., 2014). They were on average 23 years old. Mostly students from various fields of

\footnotetext{
${ }^{4} \mathrm{~A}$ pilot study has been conducted. The results suggested that there could be a significant fraction of indifferences. Therefore, this option has been implemented in the main experiment.

${ }^{5}$ Risk aversion is found to impact behavior under the DA algorithm (Klijn et al., 2013).
} 
study took part: natural sciences (28\%), economics or mathematics (19\%), law $(15 \%)$, medicine $(7 \%)$, and humanities (16\%). Sessions lasted between 58 and 76 minutes. Average earnings were 15.40 euros (min: 6.40; max: 35.50). The experiment was programmed using the software z-Tree (Fischbacher, 2007). 


\section{Results}

\subsection{Preferences for random procedures}

This paper starts the analysis by comparing preferences for random procedures. Hypothesis 1 states that individuals strictly prefer multiple to single randomization. This is consistent with the experimental evidence: If individuals have a strict preference, then they prefer multiple to single randomization.

Result 1. (Preferences for random procedures) A fraction of $23 \%$ of individuals strictly prefers multiple to single randomization. The majority of individuals (65\%) is indifferent and not willing to pay ten euro cents to choose a procedure.

Support. Figure 4 presents proportions of individuals holding strict preferences for single or multiple randomization, or indifferences. The proportion of individuals strictly preferring $M R(23 \%)$ is significantly larger than the proportion preferring $S R(12 \%)$ according to the binomial test $(N=67, p=0.007$, one-sided $)$.

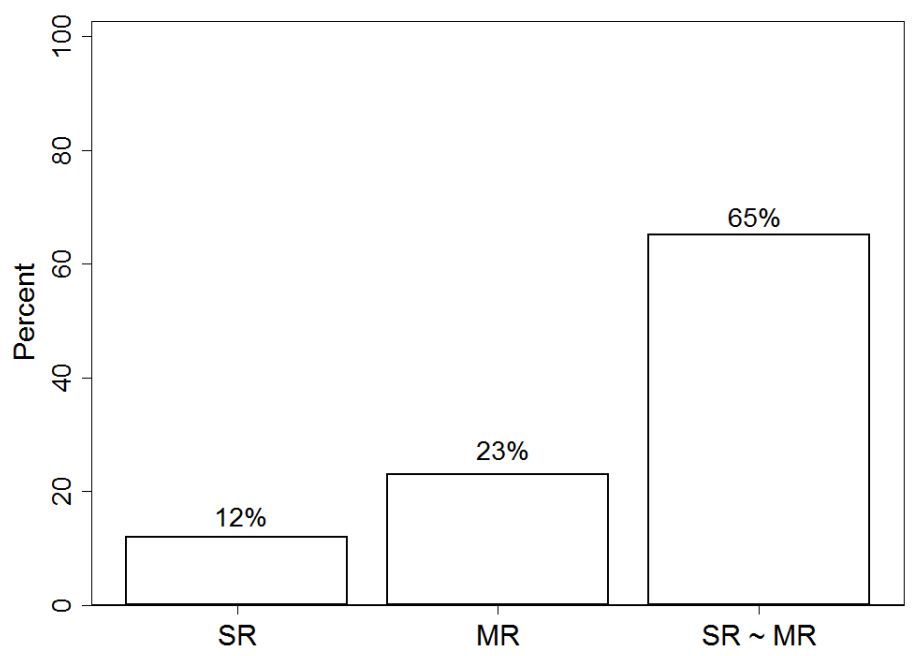

Figure 4: Proportion of individuals with preferences for single $(S R)$ and multiple $(M R)$ randomization, and indifferences $(S R \sim M R)(N=192)$.

Result 1 does not depend on the submitted preference rankings. Individuals who reported consistent rankings in tasks 1 and 2 prefer multiple $(22 \%)$ to single $(10 \%)$ randomization (binomial test, $N=29, p=0.031$, one-sided). 


\subsection{Truthful preference revelation}

Between-group differences in truthful preference revelation under $S R$ and $M R$ are analyzed on the basis of submitted preference rankings in task 1 . The reason being that preference rankings in task 2 could depend on the submitted rankings and the procedure in task 1 . Hypothesis 2 says that truth-telling rates are equal under both mechanisms. This paper finds evidence supporting the theory.

Result 2. (Truthful preference revelation) Truthful top-rank preference revelation does not differ significantly between single (69\%) and multiple (75\%) randomization; neither does truthful revelation of the entire ranking (both 47\%).

Support. Table 1 presents, in the first element of the diagonals, the proportions of truth-telling for the top-rank under $S R$ and $M R$. The proportion submitting the true top-rank does not differ significantly between $S R$ and $M R$ according to the permutation test $(N=48, p=0.363$, two-sided). For the entire ranking, the average proportion submitting the true rank order list does not differ significantly between $S R$ and $M R$ (47\%) (Mann-Whitney U, $N=48, p=0.949$, two-sided). ${ }^{6}$

Table 1: Distribution of submitted ranks (\%).

\begin{tabular}{|c|c|c|c|c|c|c|c|c|}
\hline \multirow[b]{3}{*}{ True rank } & \multicolumn{8}{|c|}{ Submitted rank } \\
\hline & \multicolumn{4}{|c|}{$S R$} & \multicolumn{4}{|c|}{$M R$} \\
\hline & 1 & 2 & 3 & 4 & 1 & 2 & 3 & 4 \\
\hline Rank 1 & 69 & 15 & 2 & 15 & \begin{tabular}{|l|}
75 \\
\end{tabular} & 17 & 2 & 6 \\
\hline Rank 2 & 23 & 50 & 20 & 7 & 18 & 49 & 22 & 11 \\
\hline Rank 3 & 5 & 15 & 74 & 6 & 5 & 18 & 69 & 8 \\
\hline Rank 4 & 3 & 14 & 4 & 79 & 2 & 13 & 7 & 78 \\
\hline
\end{tabular}

Proportions in percentages. Permutation tests are employed for individual rank comparison of the submitted ranks between $S R$ and $M R$.

No significant differences at $p<0.05$, two-sided, $N=48$.

Table 1 presents, in the diagonal elements (boxes), the percentages of truthful reporting for each rank. There are no significant differences between $S R$ and $M R$.

\footnotetext{
${ }^{6}$ The average of each group is regarded as one independent observation, resulting in $N=48$. The result for the entire ranking also holds if comparing truth-telling for each individual student preference profile (see Figure 6 in the Appendix).
} 


\subsection{Matching outcome distributions}

Reported preference rankings under the $S R$ and the $M R$ mechanism from the experiment are employed to simulate matching outcome distributions. ${ }^{7}$ Again, only reported rankings from task 1 are considered.

Result 3. (Matching distribution) The matching distributions of $S R$ and $M R d o$ not differ. Neither the proportion top choices nor the average rank received differ.

Support. Figure 5 presents cumulative distribution functions (CDF) of simulated matching outcomes using reported preferences under $S R$ and $M R$. The average proportion of individuals receiving their top choice does not differ significantly between $S R$ (47\%) and $M R$ (40\%) (permutation test, $N=48, p=0.116$, twosided). The average rank received does not differ significantly between $S R$ (1.83) and $M R$ (1.90) (Mann-Whitney $\mathrm{U}, N=48, p=0.415$, two-sided).

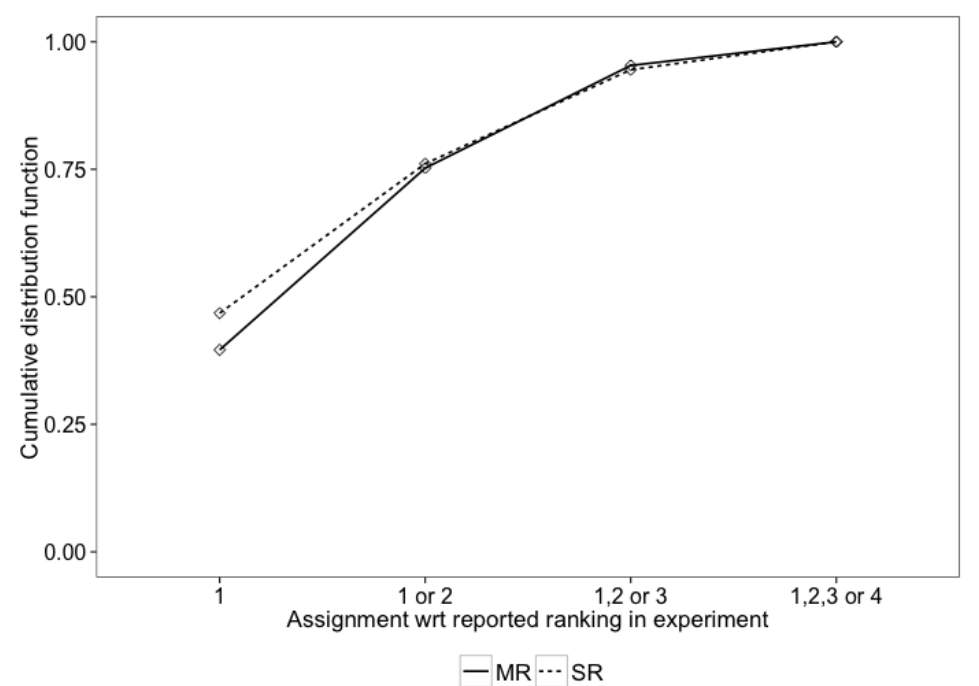

Figure 5: Cumulative matching outcome distribution based on reported preference rankings under single $(S R)$ and multiple $(M R)$ randomization.

By contrast, the matching distribution under truthful preference revelation assigns $25 \%$ to their top choice, $50 \%$ to their second preference, and $100 \%$ at least to their third preference, irrespective of the random procedure.

\footnotetext{
${ }^{7}$ Simulations are based on 10.000 independent random draws from a uniform distribution. Simulated DA outcomes are calculated using the R package by Klein (2015). Averages of the DA outcomes are calculated for each matching group.
} 


\subsection{Welfare}

Does manipulating preferences lead to welfare differences between the $S R$ and the $M R$ mechanism? Hypothesis 3 predicts that the welfare does not differ between single and multiple randomization. This is consistent with the evidence when using reported preferences from the experiment in welfare simulations.

Welfare of students is defined for each matching group as the ratio of the sum of actual payoffs given the reported preferences in the experiment and the sum of constrained efficient payoffs. The expected welfare of $S R$ and $M R$ is compared using simulations for the three schools without strict priorities, in order to let the result not depend on a particular realization of a lottery draw. Simulations are based on 10.000 randomly generated priority rankings from a uniform distribution. The mean expected welfare is calculated across matching groups for $S R$ and $M R$.

Result 4. (Expected welfare) The expected welfare does not differ significantly between the $S R$ and the $M R$ mechanism. Welfare under both mechanisms falls about $10 \%$ short of the constrained efficient level.

Support. Table 2 presents simulation results. Column 4 in reports the expected welfare under the $S R$ and $M R$ mechanism. The permutation test shows that there are no significant differences between the two mechanisms in the experiment. Welfare under preferences reported in the experiment (rows 1 and 2) is lower than the constrained efficient benchmark, but larger than the welfare obtained under random preferences (rows 3 and 4 ).

Table 2: Expected Welfare (with 10.000 Randomizations).

\begin{tabular}{lllrcc}
\hline \hline Mechanism & Preferences & N & Welfare & (SE) & Permutation test \\
\hline$S R$ & experiment & 24 & 0.889 & $(0.012)$ & $p=0.286$ \\
$M R$ & experiment & 24 & 0.916 & $(0.016)$ & \\
$S R$ & random & 24 & 0.751 & $(0.000)$ & \\
$M R$ & random & 24 & 0.751 & $(0.000)$ & \\
\hline
\end{tabular}

Note: The constrained efficient welfare level under truth-telling is 1.

Result 4 indicates that differences in preference manipulation behavior do not lead to welfare differences between both mechanisms. 


\subsection{Mechanism behind the preference for multiple randomization}

What is the mechanism behind the strict preference for multiple randomization? This analysis sheds light on the question whether fairness perceptions, biased beliefs, or risk aversion are related to the preference for multiple randomization using additional evidence from questionnaire data.

Result 5. (Fairness perception and preferences) A positive fairness judgement of $M R$ is associated with an increased likelihood of having a preference for $M R$.

Support. Table 3 presents the probit estimation results of the preference for multiple randomization. The fairness judgement of $M R$ relative to $S R$ (row 1) is in all specifications significantly positively related to the preference for $M R$. Biased beliefs about $M R$ (row 2) and risk aversion (row 3) are not significantly related to the preference for $M R$.

Table 3: Probit Regression of Preference for Multiple Randomization.

\begin{tabular}{lccccc}
\hline \hline & $(1)$ & $(2)$ & $(3)$ & $(4)$ & $(5)$ \\
\hline Fairness judgement & $0.069^{* *}$ & & & $0.068^{* *}$ & $0.082^{* * *}$ \\
& $(0.034)$ & & & $(0.034)$ & $(0.030)$ \\
Belief $M R$ & & -0.112 & & -0.124 & -0.126 \\
& & $(0.117)$ & & $(0.116)$ & $(0.092)$ \\
Risk aversion & & & -0.015 & -0.007 & -0.023 \\
& & & $(0.026)$ & $(0.025)$ & $(0.023)$ \\
Set of controls ${ }^{+}$ & No & No & No & No & Yes \\
\hline N & 67 & 67 & 67 & 67 & 67 \\
Pseudo $R^{2}$ & 0.05 & 0.01 & 0.00 & 0.07 & 0.33 \\
\hline
\end{tabular}

Dependent variable: 0 if preference for $S R$, and 1 if preference for $M R$. Average marginal effects reported. Robust standard errors in parentheses. Fairness judgement: Judgement $(M R)$ - judgement $(S R)$; Belief $M R: 1$ if belief (incentivized) that chance of highest payoff larger in $M R, 0$ otherwise.

+ Set of controls: demographics, submitted ranks in task 1 and 2, player role, order effects, field of study, math grade, set of beliefs in Appendix A.

Significance levels: ${ }^{*} p<0.10,{ }^{* *} p<0.05,{ }^{* * *} p<0.01$.

Result 5 indicates that fairness perceptions, rather than biased beliefs or risk aversion, are related to having a preference for $M R$. This result is robust to including 
indifferent subjects: The full-sample probit regression of preference for $M R$ on the fairness judgement of $M R$ indicates that there is a significant relationship $(N=192, p=0.022)$.

Fairness perceptions and biased beliefs. On average, participants judge the $M R$ mechanism (3.0) to be fairer than the $S R$ mechanism (2.7) on a Likert scale from 0 to 6 (Wilcoxon signed-rank, $N=192, p=0.003$, two-sided). Being incentivized, subjects also expect the other participants on average to judge $M R$ (3.1) to be fairer than $S R$ (2.8) (Wilcoxon signed-rank, $N=192, p<0.001$, twosided). This result indicates a robust difference in fairness perceptions between the two mechanisms.

Biased beliefs that $M R$ yields a higher chance to receive the highest payoff could potentially explain these differences in fairness judgements. This belief is elicited incentivized after the experiment. Row 2 of Table 3 shows that biased beliefs are not related to the preference for $M R$. Further, these beliefs are not correlated with fairness judgements of $M R(N=192, r=0.03, p=0.639)$. This result suggests that the fairness of a mechanism is evaluated independent of biased beliefs. 


\section{Discussion and conclusion}

This paper examines behavior under the DA mechanism with single and multiple randomization focusing on preferences for either random procedure, truthful preference revelation, and welfare. The main result clearly shows that a significant proportion of participants prefers multiple to single randomization. However, not all individuals strictly prefer one random procedure to the other. Consistent with standard expected utility theory, the majority of participants is indifferent between both random procedures. This evidence suggests that there exists a preference for multiple randomization in addition to the well-documented preference for randomization (Dwenger et al., 2014; Agranov and Ortoleva, 2015).

One mechanism behind this preference for multiple randomization is fairness perception, rather than biased beliefs or risk aversion. The behavioral theory is intended to structure the choice problem. Multiple randomization is perceived to be fairer and fairness perceptions are related to preferences for the $M R$ procedure. This relation to preferences goes beyond the previous results on fairness of random procedures (Keren and Teigen, 2010; Eliaz and Rubinstein, 2014). There is no evidence that fairness judgements are associated with biased beliefs. This implies that individuals judge multiple randomization to be fairer, although they do not expect better chances in obtaining the highest outcome from this procedure. This result is limited to beliefs about the top choice school.

Consistent with theory, truthful preference revelation is not affected by the randomization procedure. Overall, truth-telling is far below the theoretical prediction of $100 \%$. On average, $47 \%$ of participants submit their true preferences in this correlated environment. This result is in line with other experimental findings on the DA algorithm employing different environments (Chen and Sönmez, 2006; Pais and Pintér, 2008; Calsamiglia et al., 2010).

Theoretical welfare differences are ruled out by design. Consistent with the theory, this experiment finds that welfare does not differ between $S R$ and $M R$. Even though the majority of subjects does not use their weakly dominant strategies, sim-

ilar levels of truth-telling and thereby welfare results are obtained. Related works of Abdulkadiroğlu et al. (2009) and Ashlagi et al. (2015) find that single randomization yields superior welfare results. Abdulkadiroğlu et al. (2009) employ submitted preferences under one random procedure and simulate a counterfactual under the 
second procedure. In contrast, this paper uses reported preference rankings under both random procedures. Moreover, the analytical results and simulations of Ashlagi et al. (2015) are based on stochastic preferences. Importantly, and distinct from previous works, the market environment in this paper is designed in order to yield identical welfare irrespective of the randomization procedure.

In terms of external validity, the real-life effect of a preference for multiple randomization may be underestimated due to the stylized setting. The high-stakes school choice context could potentially increase concerns about the procedure. High stakes could be associated with stronger fairness perceptions. Further, increasing the number of indifferent schools could enhance fairness perceptions of multiple randomization. In turn, stronger fairness perceptions could lead to repugnance of unfair procedures. Anecdotal evidence from the NYC policy debate supports this conjecture (Pathak, 2011). NYC first implemented multiple randomization and changed it later to single randomization. Yet, this paper has shown that, even in an abstract lab environment, a significant proportion of individuals exhibits a preference for multiple randomization.

In sum, assignment mechanisms of public resources can be evaluated in terms of welfare and procedural fairness. The design of matching markets in practice often involves the choice between procedures to randomize allocation priorities. The matching literature finds that single randomization is preferable in terms of welfare (Abdulkadiroğlu et al., 2009). This paper complements these results by showing that fairness perceptions and procedural preferences speak against single randomization when welfare differences are ex ante ruled out by design. This implies that designers of such allocation procedures potentially face a trade-off between social welfare and procedural fairness perceptions.

In conclusion, this paper shows that randomization-although satisfying the "egalitarian principle" of equal chance (Elster, 1991) - is not inherently neutral with respect to procedural preferences and fairness perception. I provide evidence for a systematic distinction between a universal, single and a decentralized, multiple randomization. Random procedures as such neither guarantee equal fairness judgements nor indifference. This finding can have consequences for the social acceptability of market designs in practice. Fairness judgements and preferences point to a larger social acceptability of the multiple randomization mechanism. 


\section{References}

AbdulkadiroĞlu, A., Pathak, P. and Roth, A. E. (2005). The New York City High School Match. American Economic Review, 95 (2), 364-367.

- , - and Roth, A. E. (2009). Strategy-Proofness versus Efficiency in Matching with Indifferences: Redesigning the NYC High School Match. American Economic Review, 99 (5), 1954-78.

- and Sönmez, T. (2003). School Choice: A Mechanism Design Approach. American Economic Review, 93 (3), 729-747.

Agranov, M. and Ortoleva, P. (2015). Stochastic Choice and Preferences for Randomization. Journal of Political Economy, forthcoming.

Arnosti, N. (2016). Short Lists in Centralized Clearinghouses. mimeo.

Ashlagi, I. and Nikzad, A. (2015). What Matters in Tie-breaking Rules? How Competition Guides Design. mimeo.

- , - and Romm, A. I. (2015). Assigning More Students to Their Top Choices: A Tiebreaking Rule Comparison. mimeo.

Bock, O., Baetge, I. and Nicklisch, A. (2014). hroot: Hamburg Registration and Organization Online Tool. European Economic Review, 71, 117-120.

Bolton, G. E., Brandts, J. and Ockenfels, A. (2005). Fair Procedures: Evidence from Games Involving Lotteries. The Economic Journal, 115 (506), 1054-1076.

Bowman, D., Minehart, D. and Rabin, M. (1999). Loss Aversion in a ConsumptionSavings Model. Journal of Economic Behavior $\mathcal{E}$ Organization, 38 (2), 155-178.

Calsamiglia, C., Haeringer, G. and Klijn, F. (2010). Constrained School Choice: An Experimental Study. American Economic Review, 100 (4), 1860.

Chen, Y. and Sönmez, T. (2006). School Choice: An Experimental Study. Journal of Economic Theory, 127 (1), 202-231.

Chlass, N., Güth, W. and Miettinen, T. (2016). Purely Procedural Preferences Beyond Procedural Equity and Reciprocity. Experimental Economics, forthcoming.

De Haan, M., Gautier, P. A., Oosterbeek, H. and Van der Klaauw, B. (2016). The Performance of School Assignment Mechanisms in Practice. mimeo.

Dubins, L. E. and Freedman, D. A. (1981). Machiavelli and the Gale-Shapley Algorithm. American Mathematical Monthly, 88 (7), 485-494.

Dwenger, N., KüBler, D. F. and Weizsäcker, G. (2014). Flipping a Coin: Theory and Evidence. mimeo. 
Eliaz, K. and Rubinstein, A. (2014). On the Fairness of Random Procedures. Economics Letters, 123 (2), 168-170.

Elster, J. (1991). Local Justice: How Institutions Allocate Scarce Goods and Necessary Burdens. European Economic Review, 35 (2), 273-291.

Erdil, A. and Ergin, H. (2008). What's the Matter with Tie-Breaking? Improving Efficiency in School Choice. American Economic Review, 98 (3), 669-689.

Featherstone, C. and Niederle, M. (2014). Improving on Strategy-proof School Choice Mechanisms: An Experimental Investigation. mimeo.

Fischbacher, U. (2007). z-Tree: Zurich Toolbox for Ready-made Economic Experiments. Experimental Economics, 10 (2), 171-178.

Gale, D. and Shapley, L. S. (1962). College Admissions and the Stability of Marriage. American Mathematical Monthly, 69 (1), 9-15.

Holt, C. A. and Laury, S. K. (2002). Risk Aversion and Incentive Effects. American Economic Review, 92 (5), 1644-1655.

Kahneman, D. and Tversky, A. (1979). Prospect Theory: An Analysis of Decision under Risk. Econometrica, 47 (2), 263-291.

Keren, G. and Teigen, K. H. (2010). Decisions by Coin Toss: Inappropriate but Fair. Judgment and Decision Making, 5 (2), 83-101.

KLEIn, T. (2015). matchingMarkets: Structural Estimator and Algorithms for the Analysis of Stable Matchings. R package version 0.1-4.

Klijn, F., Pais, J. and Vorsatz, M. (2013). Preference Intensities and Risk Aversion in School Choice: A Laboratory Experiment. Experimental Economics, 16 (1), 1-22.

Köszegi, B. and Rabin, M. (2007). Reference-Dependent Risk Attitudes. American Economic Review, 97 (4), 1047-1073.

PAis, J. and Pintér, Á. (2008). School Choice and Information: An Experimental Study on Matching Mechanisms. Games and Economic Behavior, 64 (1), 303-328.

Pathak, P. A. (2011). The Mechanism Design Approach to Student Assignment. Annual Review of Economics, 3 (1), 513-536.

- and Sethuraman, J. (2011). Lotteries in Student Assignment: An Equivalence Result. Theoretical Economics, 6 (1), 1-17.

Roth, A. E. (1982). The Economics of Matching: Stability and Incentives. Mathematics of Operations Research, 7 (4), 617-628.

Rubinstein, A. (2002). Irrational Diversification in Multiple Decision Problems. European Economic Review, 46 (8), 1369-1378. 


\section{Appendix for online publication}

\section{A Tables and figures}

Table 4: Fairness judgements.

\begin{tabular}{lccccc}
\hline \hline Variable & Sample & N & Mean & $(\mathrm{SE})$ & $\begin{array}{c}\text { P-value } \\
\text { signed-rank }\end{array}$ \\
\hline$S R$ & full & 192 & 2.729 & $(0.108)$ & 0.003 \\
$M R$ & full & 192 & 3.010 & $(0.113)$ & \\
Belief $S R$ & full & 192 & 2.813 & $(0.085)$ & $<0.001$ \\
Belief $M R$ & full & 192 & 3.068 & $(0.088)$ & \\
$S R$ & reduced & 44 & 2.727 & $(0.196)$ & 0.001 \\
$M R$ & reduced & 44 & 3.545 & $(0.224)$ & \\
Belief $S R$ & reduced & 44 & 2.841 & $(0.166)$ & 0.002 \\
Belief $M R$ & reduced & 44 & 3.432 & $(0.185)$ & \\
\hline
\end{tabular}

Fairness judgements are measured using a 7 point Likert scale $(0-6)$.

$S R$ and $M R$ present fairness judgements about the respective mechanism.

Belief $S R$ and $M R$ are incentivized beliefs about average judgement of others.

Reduced sample includes only participants with preference for $M R$.

P-values of two-sided Wilcoxon signed-rank test are reported. 
Table 5: Belief distributions.

\begin{tabular}{|c|c|c|c|c|c|c|}
\hline \multirow[t]{2}{*}{ Variable } & \multirow[t]{2}{*}{$\mathrm{N}$} & \multicolumn{3}{|c|}{ Distribution (\%) } & \multirow{2}{*}{$\begin{array}{c}\mathrm{N} \\
(S R \& M R)\end{array}$} & \multirow{2}{*}{$\begin{array}{c}\text { P-value } \\
\text { binomial test }\end{array}$} \\
\hline & & $S R$ & $M R$ & $S R \sim M R$ & & \\
\hline Preference & 192 & 11.98 & 22.92 & 65.10 & 67 & 0.014 \\
\hline Belief preference & 192 & 19.27 & 27.08 & 53.65 & 89 & 0.140 \\
\hline Belief procedure & 192 & 16.67 & 26.04 & 57.29 & 82 & 0.060 \\
\hline
\end{tabular}

Preference is the benchmark distribution of preferences reported in figure 4.

Belief preference is the incentivized belief about the average preferences of others.

P-values of two-sided binomial test $(S R=M R)$ are reported $(N$ of $S R \& M R)$.

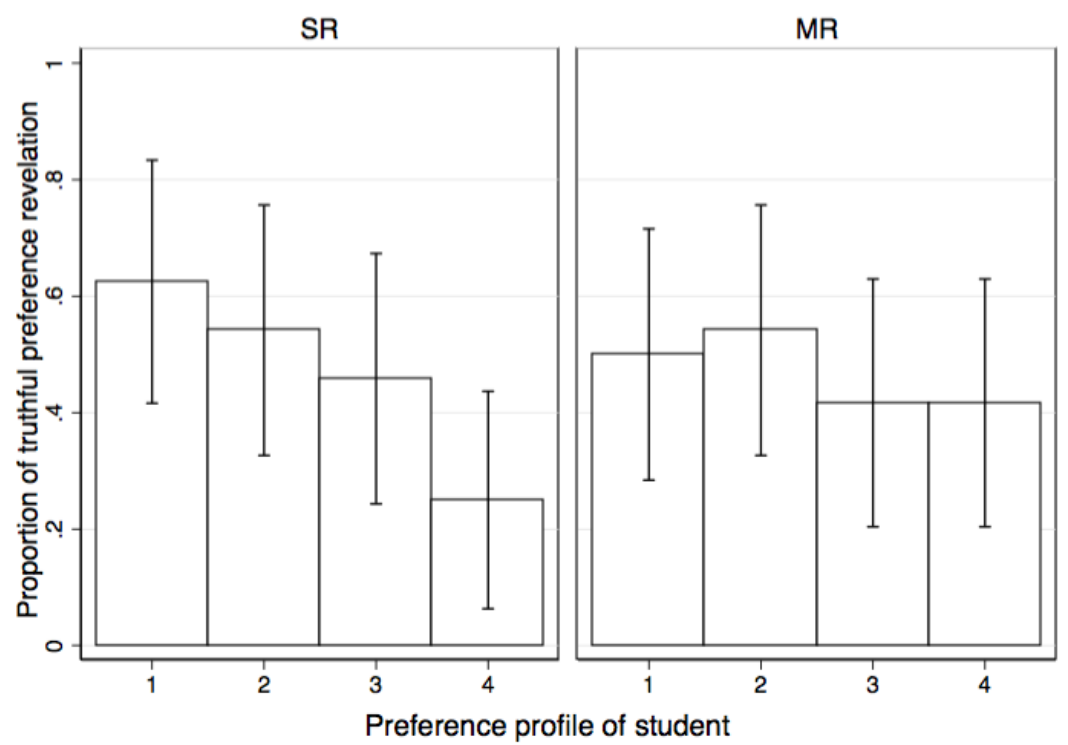

Figure 6: Proportions of truthful preference revelation by student preference profile under $S R$ and $M R$ (error bars present $95 \%$ confidence intervals). 


\section{B Proof of Proposition 2}

Proposition 2. The expected utility of the simple lottery $L$ is equivalent to the expected utility of the compound lottery $\mathcal{L}, E U(L)=E U(\mathcal{L})$.

Proof. The proof is shown based on the example used in the experiment. There are four students and four schools. Let $L=\left(q_{1}, \ldots, q_{4}\right)$ be the simple lottery and let $\mathcal{L}=\left\{L_{s_{1}}, \ldots, L_{s_{4}} ; \alpha_{1}, \ldots, \alpha_{4}\right\}$ be the four-stage compound lottery of four simple lotteries. Lottery $L_{s_{4}}$ is degenerate and yields the priority ranking with certainty.

First, note that there are 4 ! possible priority orderings over the number of students $N=4$ of the set of students $I=\{1, \ldots, 4\}$ at each school $s \in S$. Lotteries are drawn from a uniform distribution. That is, each ordering is equally likely with probability $\frac{1}{4 !}$ at school $s$. All $(4 !)^{3}$ priority orderings are equally likely. Let the preference relation satisfy axioms of expected utility theory (EU).

Example. Consider the following environment from the experiment:

$$
\begin{aligned}
& s_{1} \mathrm{P}_{i_{1}} s_{2} P_{i_{1}} s_{3} P_{i_{1}} s_{4} \quad i_{1} \sim_{s_{1}} i_{2} \sim_{s_{1}} i_{3} \sim_{s_{1}} i_{4}
\end{aligned}
$$

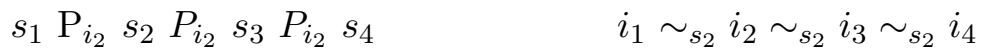

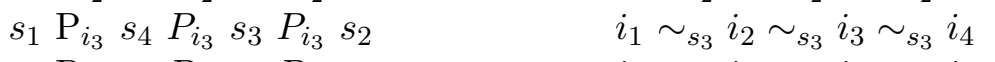

$$
\begin{aligned}
& \begin{array}{lllllll}
s_{1} & \mathrm{P}_{i_{4}} & s_{4} & P_{i_{4}} & s_{3} & P_{i_{4}} & s_{2}
\end{array} \quad, \quad i_{1} \succ_{s_{4}} i_{2} \succ_{s_{4}} i_{3} \succ_{s_{4}} i_{4}
\end{aligned}
$$

Simple lottery. Assuming truthful preference revelation of the preference profiles $P_{i}$, the expected utility of the simple lottery $L$ is calculated as follows

$$
\begin{aligned}
E U(L) & =\sum_{n=1}^{4} q_{n} u\left(\pi_{i, s_{m}}\right) \\
i=1,2: \quad E U(L) & =\frac{1}{4} u\left(\pi_{i, s_{1}}\right)+\frac{1}{2} u\left(\pi_{i, s_{2}}\right)+\frac{1}{4} u\left(\pi_{i, s_{3}}\right), \\
i=3: \quad E U(L) & =\frac{1}{4} u\left(\pi_{i, s_{1}}\right)+\frac{3}{4} u\left(\pi_{i, s_{3}}\right), \\
i=4: \quad E U(L) & =\frac{1}{4} u\left(\pi_{i, s_{1}}\right)+\frac{3}{4} u\left(\pi_{i, s_{3}}\right),
\end{aligned}
$$

where $q_{n}$ is the probability of getting the payoff; $\pi_{i, s_{m}}$ is the payoff of student $i$ receiving the seat at school $s$.

Reduction of the four-stage compound lottery. Since every simple lottery in the compound lottery is drawn from the same uniform probability distribution and using the Independence axiom of EU, the compound lottery can be reduced to the simple lottery:

$$
E U(L)=q_{1} u\left(\pi_{i, s_{1}}\right)+q_{2} u\left(\pi_{i, s_{2}}\right)+q_{3} u\left(\pi_{i, s_{3}}\right)+q_{4} u\left(\pi_{i, s_{4}}\right)=E U(\mathcal{L})
$$

Since the compound lottery $(\mathcal{L})$ can be reduced to the simple lottery $(L)$, both are equal in expected utility: $E U(L)=E U(\mathcal{L})$. 


\section{Instructions}

Notes: Part one consists of instructions on a handout. Part two is displayed on the computer screen during the experiment. Quiz questions are based on Klijn et al. (2013).

Part 1

Welcome to the experiment! You are going to take part in an economic study financed by the Max Planck Society. You will receive a show-up fee of 4 euros. Additionally, you will be able to earn a substantial amount of money. It is therefore crucial that you read these explanations carefully. Your payment depends on your decisions, on the decisions of the other participants, and on chance. The present instructions are identical for all participants.

From now on it is prohibited to use electronic devices. During the experiment there shall be absolutely no communication between participants. If you have any questions, please raise your hand. We will then come over to you. Any violation of these rules means you will be excluded from the experiment and from any payments.

During the experiment we will calculate in points. The total number of points you earn in the course of the experiment will be transferred into euro at the end, at a rate of

$$
1 \text { euro }=20 \text { points. }
$$

The procedure and payment details are described below. Following the instructions, we ask you to answer a quiz. This shall help you to understand the decision situation.

\section{Procedure \\ - Task 1 \\ - Task 2 \\ - Questionnaire \\ - Payment}

The experiment consists of three parts: Task 1, task 2, and the questionnaire. You will receive precise instructions for task 1 and task 2 on your computer screen after the experiment has begun. At the beginning of the experiment, all participants are randomly assigned to groups of four. You will not get to know the identity of the other participants in your group. You stay in the same group during both tasks.

Your cash payment at the end of the experiment consists of the following parts. You receive 4 euros show-up fee. In the end, one of both tasks (either 1 or 2) is randomly determined and payd out to you. Additionally, you can earn money by answering the questionnaire. 


\section{Decision Situation}

In tasks 1 and 2, we simulate a procedure that assigns positions to applicants. You and the other participants are applicants. Within each group, you are randomly assigned the role of an applicant. This role remains the same in both tasks.

Applicants use the procedure of a central clearinghouse to apply for positions. The computer determines who gets which position. In the following, payment table, priority lists, and the ranking are explained. Consequently, the procedure of allocating positions using this information is described.

There are four applicants (1,2,3, and 4) and four positions (W,X,Y, and Z). Every position accepts only one applicant. The following payment table determines your points during the experiment.

Payment table.

\begin{tabular}{rllll}
\hline Points & Applicant 1 & Applicant 2 & Applicant 3 & Applicant 4 \\
\hline 280 points & Position W & Position W & Position W & Position W \\
200 points & Position X & Position X & Position Z & Position Z \\
120 points & Position Y & Position Y & Position Y & Position Y \\
40 points & Position Z & Position Z & Position X & Position X \\
\hline
\end{tabular}

In this payment table you can see how many points each applicant receives for each assigned position. This table is equivalent in both tasks. For instance, if applicant 1 is assigned position $\mathrm{W}$ in the allocation procedure, then he receives 280 points. If applicant 1 receives position $\mathrm{X}$, then he receives 200 points; for position $\mathrm{Y}$ he receives 120 points and for position $\mathrm{Z}$ he receives 40 points. Positions have the following priority lists over the applicants in the experiment.

Priority lists.

\begin{tabular}{|c|c|c|c|}
\hline Priority & Position W Position X & Position Y & Position Z \\
\hline Highest priority & & & Applicant 1 \\
\hline Second-highest priority & & & Applicant 2 \\
\hline Third-highest priority & & & Applicant 3 \\
\hline Lowest priority & & & Applicant 4 \\
\hline
\end{tabular}

In this table, you can see that applicant 1 is on top of position Z's priority list. Position Z has highest priority for applicant 1, followed by applicant 2 with second-highest, applicant 3 with third-highest, and applicant 4 with lowest priority. Priority means, for example, that applicant 1 is favored by position $\mathrm{Z}$ compared to the other applicants. Please note that during the experiment only the priority list of position $\mathrm{Z}$ is given. You receive more information about the priority lists of positions $\mathrm{W}, \mathrm{X}$, and $\mathrm{Y}$ on your computer screen during the experiment. 


\section{Your Decision}

In each task, you will make a decision about a ranking of positions. You may submit any ranking. All positions have to be listed. Rank 1 means the top rank, rank two the second-highest, rank 3 the third-highest, and rank 4 the lowest rank.

Every applicant submits a ranking of positions. Given these rankings and the priority lists, positions are assigned in the central allocation procedure. The final payment is made according to the payment table.

\section{Description of the Allocation Procedure}

1. Every applicant applies for his top rank position.

2. Every position compares applicants and temporarily accepts the applicant with highest priority on its priority list. All others are rejected.

3. If an applicant is rejected, then he applies for the position with the second-highest rank on his submitted ranking.

4. If a position gets new applications (previously rejected applicants at other positions), then it considers both new applicants and temporarily accepted applicant. The position compares new participants with the temporarily accepted participant and temporarily accepts the one with highest priority. All others are rejected.

5. This allocation procedure is repeated until no applicant is rejected anymore. Every applicant receives the position that accepts his application in the end. 


\section{An Example}

Consider for illustration purposes the following example. There are three applicants $(1,2$, and 3$)$ and three positions (A, B, and C). Assume that the applicants submitted the following rankings:

$$
\begin{array}{llll}
\text { Applicant 1: } & \operatorname{rank} 1=\mathrm{B} & \operatorname{rank} 2=\mathrm{C} & \operatorname{rank} 3=\mathrm{A} . \\
\text { Applicant 2: } & \operatorname{rank} 1=\mathrm{C} & \operatorname{rank} 2=\mathrm{A} & \operatorname{rank} 3=\mathrm{B} . \\
\text { Applicant 3: } & \operatorname{rank} 1=\mathrm{B} & \operatorname{rank} 2=\mathrm{C} & \operatorname{rank} 3=\mathrm{A} .
\end{array}
$$

Important: These sample rankings are chosen arbitrarily and only serve illustrational purposes. They provide no guidance for your decision-making in the experiment!

Assume the following priority lists. In this example, all priority lists are given. In the experiment, only one priority list is given.

Example: Priority lists.

\begin{tabular}{lccc}
\hline Priority & Position A & Position B & Position C \\
\hline Highest priority & 2 & 2 & 1 \\
Second-highest priority & 3 & 1 & 3 \\
Lowest priority & 1 & 3 & 2 \\
\hline
\end{tabular}

You can see for example in the rankings that applicant 1 submitted position B at the top rank. Position $\mathrm{C}$ is on the second-highest rank and position $\mathrm{A}$ is on the lowest rank. Further, you can see from the priority lists that position A has highest priority for applicant 2 followed by applicants 3 and 1. 


\section{Questions about the Example}

Please complete the following sentences.

1. In round one of the allocation procedure, every applicant applies for his top rank position. That is, applicant 1 applies for position __ applicant 2 for position __ and applicant 3 for position _. Based on these three applications, every position temporarily accepts one applicant and rejects all others according to their priority. Position B compares applicants 1 and 3 according to priority. Then, position B temporarily accepts applicant and rejects applicant . Position $\mathrm{C}$ temporarily accepts applicant

2. In round two, all previously rejected applicants apply for the position with the second highest rank. That is, applicant 3 applies for position __ Now, positions compare new applicants with the temporarily accepted ones from round one. Position C compares applicant 2 with 3 . Applicant 3 has a higher priority then applicant 2. Then, position C temporarily accepts applicant and rejects applicant

3. In round three, every applicant rejected in round two applies for the position with the next-highest rank in his ranking. That is, applicant applies for position . Since this position has not been assigned yet, all applicants are now assigned one position. The central allocation procedure is terminated.

4. The final allocation of positions is as follows.

Applicant receives position $\mathrm{A}$.

Applicant receives position $\mathrm{B}$.

Applicant receives position $\mathrm{C}$.

5. Are both tasks 1 and 2 selected to be payoff relevant in the end? Answer:

Do you have any questions? If this is the case, then please raise your hand. We will answer your questions individually. Thank you for participating in this experiment! 
Part 2 [for order $S R, M R$ ]

Screenshot $S R$.

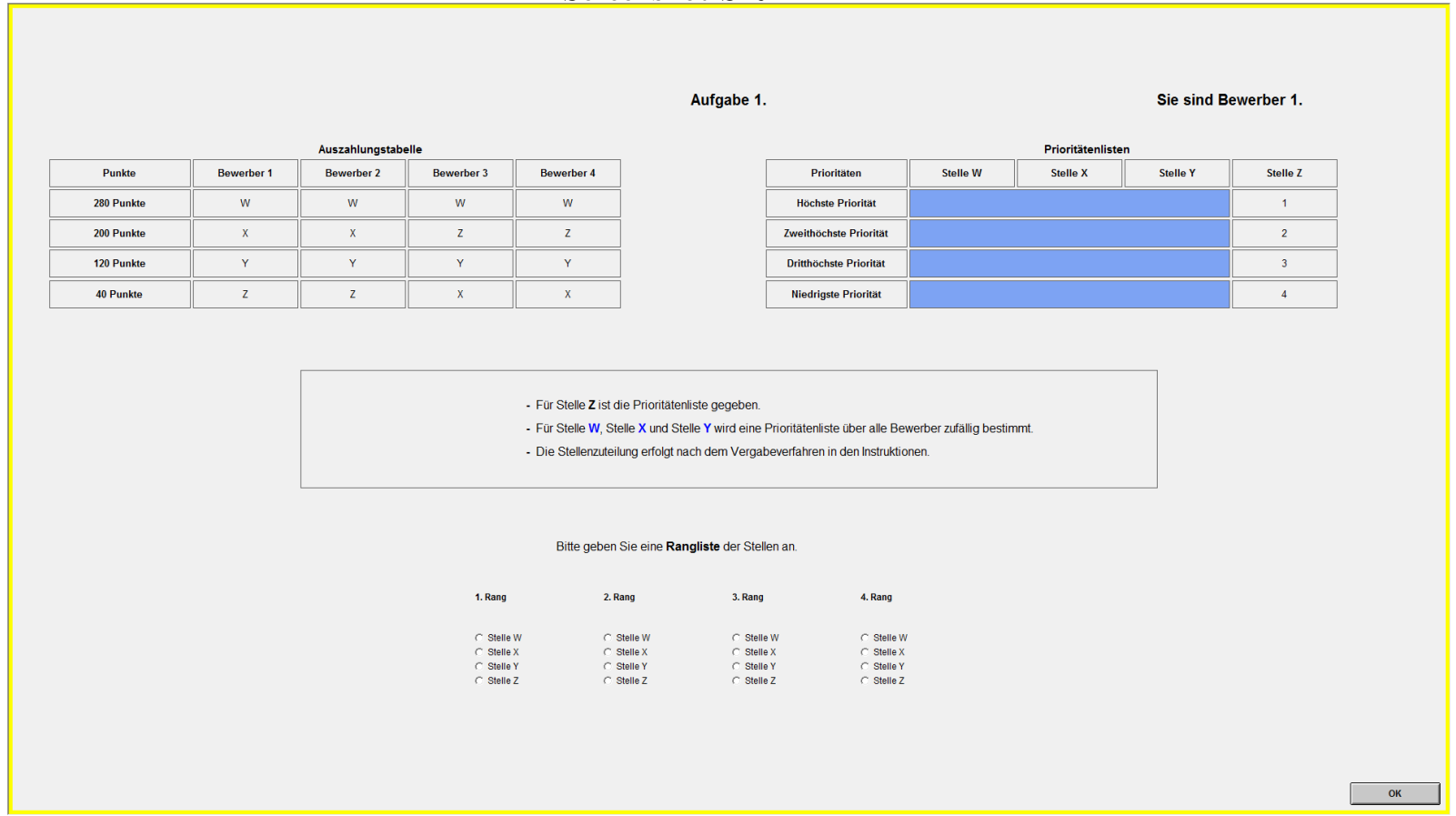

Task 1 [SR description]

- The priority list of the four applicants is given for position $\mathbf{Z}$.

- One joint priority list of all applicants will be randomly drawn for position $\mathbf{W}$, position $\mathbf{X}$, and position $\mathbf{Y}$.

In the end, the priority list is randomly drawn by the computer.

Any combination of applicants is equally likely.

Then, assignment of positions takes place according to the procedure in the instructions. 
Screenshot $M R$.

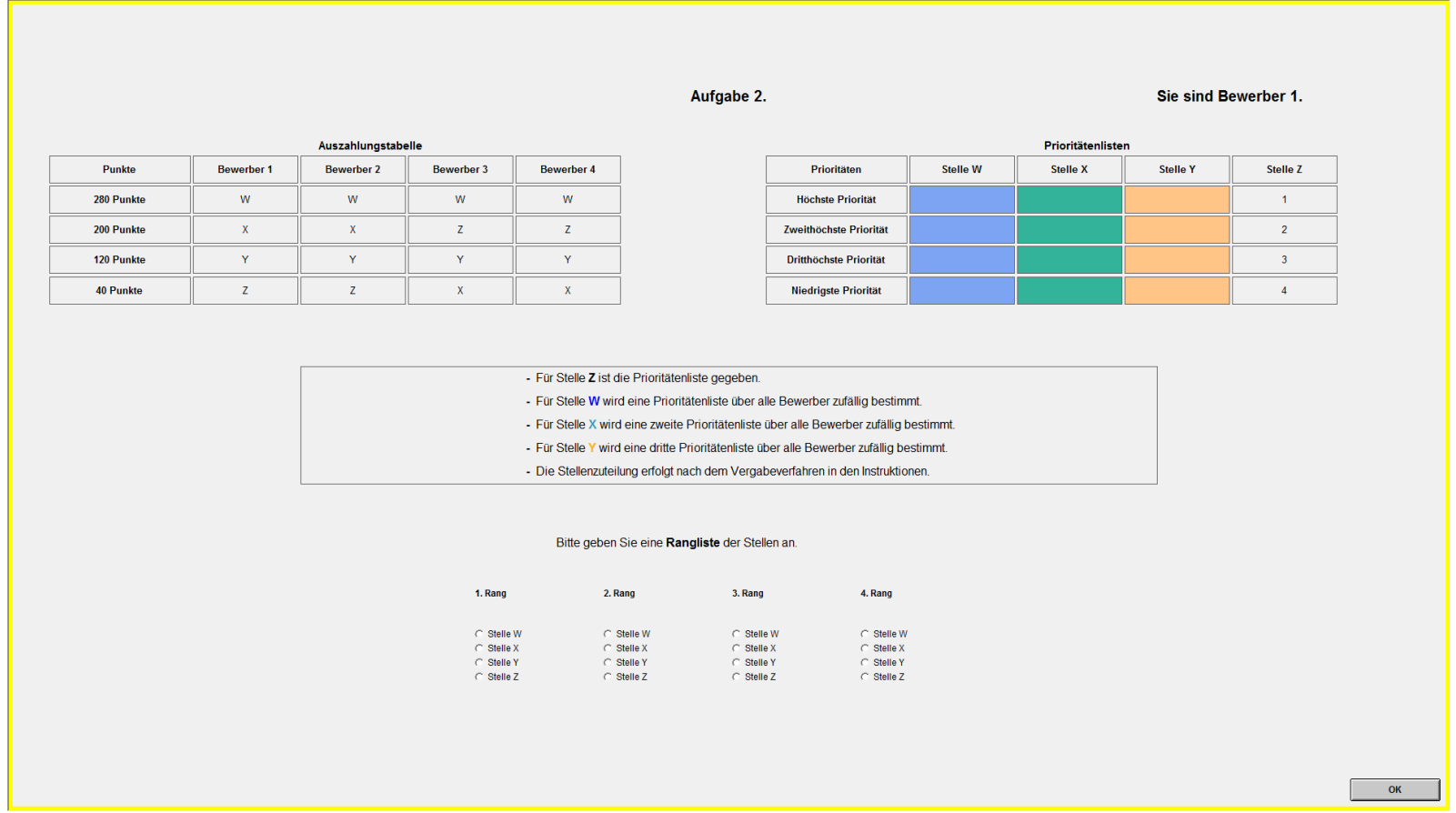

Task $2[M R$ description $]$

- The priority list of the four applicants is given for position $\mathbf{Z}$.

- One priority list will be randomly drawn for position $\mathbf{W}$.

- One priority list will be randomly drawn for position X.

- One priority list will be randomly drawn for position $\mathrm{Y}$.

In the end, the priority lists are randomly drawn by the computer.

Any combination of applicants is equally likely.

Then, assignment of positions takes place according to the procedure in the instructions. 
Question 1 [Preferences for random procedures]

Now you have the chance additionally to earn up to 280 points.

- You just made decisions about rankings in task 1 and task 2.

One task has been randomly determined to be payoff-relevant for the previous part.

- Now you can earn additional money with your decisions from one of the tasks. You can decide for yourself which task should determine your additional payment. If you do not decide, then the computer randomly determines one task.

- If you choose one of the tasks, then you pay 2 points for your decision option. If you do not decide, you do not pay 2 points.

I choose task 1.

I choose task 2.

I do not choose. 